\title{
Evolution of Heterogeneous Cellular Automata in Fluctuating Environments
}

\author{
David Medernach $^{1, *}$, Simon Carrignon ${ }^{2,3}$, René Doursat $^{4}$, Taras Kowaliw, Jeannie Fitzgerald ${ }^{1}$ and Conor Ryan ${ }^{1}$ \\ ${ }^{1}$ Biocomputing and Developmental Systems (BDS), University of Limerick, Ireland \\ ${ }^{2}$ Barcelona Supercomputing Center (BSC), Catalonia, Spain \\ ${ }^{3}$ ICREA-Complex Systems Lab, Universitat Pompeu Fabra, Barcelona, Catalonia, Spain \\ ${ }^{4}$ Informatics Research Centre (IRC), Manchester Metropolitan University, UK \\ *david.medernach@ul.ie
}

\begin{abstract}
The importance of environmental fluctuations in the evolution of living organisms by natural selection has been widely noted by biologists and linked to many important characteristics of life such as modularity, plasticity, genotype size, mutation rate, learning, or epigenetic adaptations. In artificial-life simulations, however, environmental fluctuations are usually seen as a nuisance rather than an essential characteristic of evolution. HetCA is a heterogeneous cellular automata characterized by its ability to generate open-ended long-term evolution and "evolutionary progress". In this paper, we propose to measure the impact of different types of environmental fluctuations in HetCA. Our results indicate that environmental changes induce mechanisms analogous to epigenetic adaptation or multilevel selection. This is particularly prevalent in two of the tested fluctuation schemes, which involve a round-robin inhibition of certain cell types, where phenotypic selection seems to occur.
\end{abstract}

\section{Introduction}

In natural evolution, environmental changes may include cyclic events such as seasonal changes and the daily alternation of light and darkness, occasional changes such as the appearance of new predators and the potential for new food sources, or more radical modifications such as environmental stresses induced by climate transitions.

Since the work of Levins (1968) on evolution in changing environments and more recent attempts to integrate "epigenetic inheritance systems" (EIS) (Heard and Martienssen, 2014), developmental or "evo-devo" processes (Müller, 2007) and "niche construction" effects (Laland et al., 2016), emphasis has been put on the importance and impact of changes on the course of the evolution of living organisms by natural selection. Through such works, environmental fluctuations have been linked to many central properties and mechanisms of evolution, among which the most discussed examples are modularity, plasticity (West-Eberhard, 2005), genome size, mutation rates, and evolvability.

For Jablonka et al. (2014), changing environments unmask variations in the capacity of individuals to make adjustments to new conditions, therefore promoting plasticity and multilevel selection. These authors contend that "For a lineage in a constantly changing environment, switching among several alternative heritable states was probably an advantage. While cells in one state survived in one set of conditions, those in other states did better in different circumstances." (ibid., p. 318). In the same line of thought, continually varying or cyclic conditions might also explain the origin of EIS, as "epigenetic mutations" are more reversible and occur more frequently than genetic ones. To illustrate this notion, Lachmann and Jablonka (1996) modeled the effects of oscillating variations, such as seasonal or daily cycles, on phenotypical inheritance. Their model predicts correctly that when the environmental cycle is longer than the reproductive cycle, while remaining relatively short otherwise, heritable variations produced by non-DNA inheritance systems are likely to be observed.

In parallel to biological research, a number of studies in artificial life, especially evolutionary robotics (Floreano and Urzelai, 2000), have also investigated environmental variations, some of them explicitly defining the environment as a driving evolutionary force (Bredeche and Montanier, 2012). Others, such as Lipson et al. (2002), showed a correlation between the modularity and the rate of change of external resources, while Yu (2007) observed that populations exploit neutrality to cope with environmental fluctuations and can evolve a type of evolvability under two alternating objective functions. Both of these simulation works relied on genetic programming (GP) and explicit fitness functions.

We wish to study the effects of such fluctuations in a model closer to the living world without using an explicit objective function, and determine if these fluctuations promote phenotypic selection over genotypic selection. To that goal, we propose an open-ended experimental setup allowing us to systematically and quantitatively measure the influence of cyclic environmental fluctuations on the course of the evolution of cellular automata (CA). We show that such fluctuations lead to the emergence of processes similar to those exhibited by EIS.

The paper is organized as follows. First the general mechanisms of our Heterogeneous Cellular Automata (HetCA) model are explained. Then, the implementation of environ- 
mental fluctuations in HetCA is described. Next, we specify the computational setup used to study environmental fluctuations. This is followed by a report on the experimental results and a discussion of their implications. Finally, we propose a qualitative analysis and a conclusion.

\section{The HetCA Model}

HetCA (Medernach et al., 2013) is based on classical twodimensional CA with several additional features: cells follow a heterogeneous transition function, i.e. one which depends on their location, inspired by linear genetic programming (LGP); they can also fall into special decay and quiescent states; and there is a notion of genetic transfer of transition functions (i.e. genotypes) between adjacent cells. Decay and quiescent cells do not possess a genotype; all other cells do, and are called living. There are 5 different living states: quiescent cells can acquire a genotype from any nearby living cell and therefore become living in turn; decay cells cannot, but become quiescent after a number of consecutive iterations comprised between 375 and 1,875 after decay (Fig. 1). Living cells always automatically turn into decay after 7 consecutive iterations spent in any one or several living states (tracked by an "age" counter). Further details about the HetCA model can be found in (Medernach et al., 2013).

We showed that HetCA could exhibit long-term phenotypic dynamics, a high variance over very long runs, greater behavioral diversity than classical CA, and "evolutionary progress" (Shanahan, 2012) on three criteria: robustness, size and density of the genotype (Medernach et al., 2015).

Finally, while there is a lasting debate over the units of selection in evolutionary biology since the origins of the field, including genotype selection, phenotype selection, epigenetic selection, behavioral selection, multilevel selection, group selection, and so on (Lloyd, 2012; Okasha, 2006), several of them are potentially included in HetCA. There is genotypic selection of the transition rules, but also phenotypic selection of cell groups able to replicate patterns, such as the ones found in the Game of Life. This point is important when one is interested in environmental fluctuations because, as mentioned in the introduction, we anticipate that the existence of frequent environmental fluctuations will promote phenotypic selection over genotypic selection.

\section{Experimental Setup}

As in the previous version of HetCA (Medernach et al., 2013), the genotype of an individual consists of its transition rules encoded in a custom CA-LGP program using the function set listed in Table 1. Such a program maps the space of neighborhood states to a new cell state, while providing an evolvable representation framework based on an alphabet of elementary functions. Individual genotypes are modified by micro-mutations (change in one component of a statement)

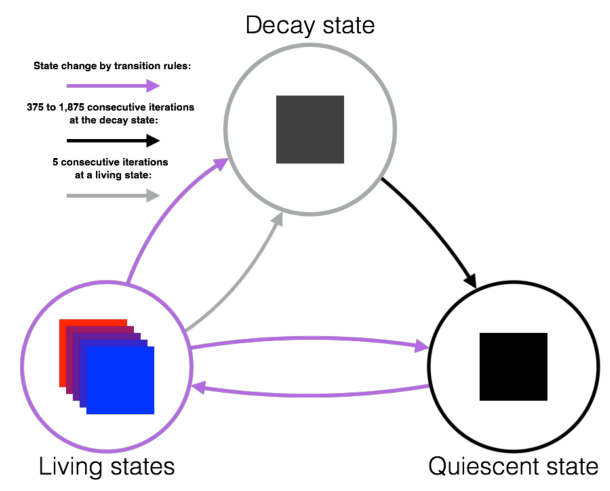

Figure 1: A cell's life cycle between states.

Table 1: Function set.

\begin{tabular}{lc}
\hline operator & action on inputs $(x, y)$ \\
\hline abs & $|x|$ \\
plus & $x+y$ \\
delta & 1, if $|x-y|<1 / 10^{4} ; 0$ o.w. \\
dist & $|x-y|$ \\
inv & $1-x$ \\
inv2 & $\operatorname{safeDiv}(1, x)$ \\
magPlus & $|x+y|$ \\
max & $\max \{x, y\}$ \\
min & $\min \{x, y\}$ \\
safeDiv & $x / y$ if $|y|>1 / 10^{4} ; 1$ o.w. \\
safePow & $x^{y}$, if $\operatorname{defined} ; 1$ o.w. \\
thresh & 1, if $x>y ; 0$ o.w. \\
times & $x y$ \\
zero & 1, if $|x|<1 / 10^{4} ; 0$ o.w. \\
\hline
\end{tabular}

and macro-mutations (addition or removal of an entire statement) of the corresponding CA-LGP programs.

Previously, the new genotype $g$ of a living cell $c$ during genetic transfer was chosen randomly among candidate genotypes according to a uniform distribution. To be candidate, a genotype had to come from a living cell in $c$ 's immediate neighborhood (von Neumann). In the present study, to introduce environmental variations we vary the likelihood of propagation of a genotype according to its cell state $s(c)$. In this new setup, the probability $P(c)$ of a candidate genotype to be selected becomes $P(c)=K(s(c)) / \sum_{i=1}^{n} K\left(s\left(c_{i}\right)\right)$, where $K(s)$ is the state distribution and $n$ the number of neighboring candidate genotypes, i.e. 4. Thus, an environment $E$ is characterized by the propagation probabilities of the 5 possible living states: $E=\left\{K\left(s_{1}\right), \ldots, K\left(s_{5}\right)\right\}$. To mimic environmental fluctuations we initialize the simulation with $K\left(s_{k}\right)=1$ for all $k \in[1,5]$, then regularly modify those values every $f$ iterations starting from iteration 3,000 of the CA. We introduce three types of environmental fluctuations (Table 2):

Short-cycle fluctuations $(S c F)$ consist of alternating between two opposite environments, $\{0,0,1,1,1\}$ and $\{1,1,1,0,0\}$, every $f$ iterations of the CA. We set $f=100$ to remain within the range of frequency described by Lipson et al. (2002) and Yu (2007). Here we consider that a successful reproductive cycle for a cell involves passing through the 
Table 2: Stable and fluctuating environments.

\begin{tabular}{lcccl}
\hline Name & Short Name & Cycles & Transitions & Environment list: propagation probabilities $E=\left\{K\left(s_{1}\right), \ldots, K\left(s_{5}\right)\right\}$ of the living types \\
\hline Stable environment & (SE) & NA & NA & $\{1,1,1,1,1\}$ \\
Short-cycle fluctuations & (ScF) & 100 & 1 & $\{0,0,1,1,1\},\{1,1,1,0,0\}$ \\
Light fluctuations & (LF) & 5,000 & 1 & $\{1,1,1,1,0\},\{1,1,1,0,1\},\{1,1,0,1,1\},\{1,0,1,1,1\},\{0,1,1,1,1\},\{1,1,1,1,1\}$ \\
Strong fluctuations & (SF) & 5,000 & 1 & $\{0,0,1,1,1\},\{1,1,1,0,0\},\{0,1,0,1,1\},\{1,0,1,1,0\},\{0,1,1,0,1\},\{1,1,0,1,0\}$, \\
& & & & $\{1,0,1,0,1\},\{0,1,1,1,0\},\{1,0,0,1,1\},\{1,1,0,0,1\},\{1,1,1,1,1\}$ \\
\hline
\end{tabular}

Table 3: HetCA parameters.

\begin{tabular}{lc}
\hline Parameter & Value \\
\hline Number of living states & 5 \\
Successive living iterations before decay & 7 \\
Number of iterations during decay & 375 to 1,875 \\
Direct transition to decay & enabled \\
Size of the grid & $500 \times 500$ \\
Grid boundaries & toroidal grid \\
Transition Rule (TR) & CA-LGP \\
Maximum TR size & 50 statmts \\
Genotype copy neighborhood & v. Neum. (4) \\
Transition rule neighborhood & Moore $(8)$ \\
\hline
\end{tabular}

quiescent state. This should take between 2 iterations (alternating between quiescent and living) and 7 iterations (after which a living cell decays and can no longer receive a genotype for a long period of time).

Light fluctuations ( $L F)$ consist of alternating between 6 different environments every $f=5,000$ iterations. The first 5 environments each prohibit a different living state from spreading its genotype; the last one gives an equal chance to all living states.

Strong fluctuations $(S F)$ consist of alternating between 11 environments every $f=5,000$ iterations. The first 10 environments each prohibit a different pair of living states from spreading their genotypes; the last one gives an equal chance to all pairs.

The rationale behind $\mathrm{ScF}$ is their analogy with circadian rhythms in certain bacteria. The idea is to mimic the highly regular cycles during which these organisms have enough time to reproduce repeatedly. LF, by contrast, are more similar to seasonal fluctuations, while SF resemble ecological crises. However, owing to the variety of both biological temporal rhythms and reproductive cycles, the relevance of these analogies remains limited.

\section{Simulations}

For each one of the three types of environmental fluctuations and the stable (non-fluctuating) environment (SE) we performed 50 simulations. This produced a total of $4 \times 50=$ 200 runs. Each cell of the CA was initialized in a random state, then each cell in one of the 5 living states was initialized with an individual randomly generated genotype. Each run lasted 500,000 iterations under the parameters listed in Table 3.

\section{Genotype Size}

We used the number of program statements $n_{\text {prog }}$ as a measure of genotype size and computed the average size of all current genotypes of a run every 2,500 iterations. We then reported the average and standard error of the mean (SEM) among all 50 runs sharing the same settings.

\section{Phenotype Comparison}

If environmental changes led to the emergence of phenotypic selections (similar to the EIS) using easily reversible phenotypic mutations (Jablonka et al., 2014), then phenotypes from different individuals of the same lineage observed while environmental conditions are similar should stay relatively close, even though individuals from their lineage evolved in other environmental conditions between these measures. By contrast, if the adaptation to each environmental change was done exclusively through the selection of classical, irreversible genotypic mutations, these phenotypes should be quite different, despite the potential evolutionary convergence. We developed a metric to measure phenotypic proximity between two iterations of the CA. To do this we simply used the distributions of living cells over the living states. Thus the phenotypic difference $\sigma$ between two iterations $t_{1}$ and $t_{2}$ was calculated as follows:

$$
\sigma\left(t_{1}, t_{2}\right)=\sum_{k=1}^{5}\left|\frac{N\left(s_{k}, t_{1}\right)}{N\left(t_{1}\right)}-\frac{N\left(s_{k}, t_{2}\right)}{N\left(t_{2}\right)}\right|
$$

where $N\left(s_{k}, t\right)$ is the number of cells in living state $s_{k}$ at iteration $t$ and $N(t)=\sum_{k=1}^{5} N\left(s_{k}, t\right)$ is the total number of living cells at $t$.

Every 5,000 iterations of the CA we performed two phenotypic comparisons between the current iteration $t_{1}=t$ and an iteration in the past, $t_{2}=t-\Delta t$. In one scenario, the temporal distance $\Delta t$ was a multiple of the periodicity $f$, so that we compared two similar environments: $E\left(t_{1}\right)=E\left(t_{2}\right)$. We chose $\Delta t=60,000$ in the SE, ScF and LF cases and 55,000 in the SF case. In another scenario, we introduced an additional single-period shift such that we compared two dissimilar environments: $E\left(t_{1}\right) \neq E\left(t_{2}\right)$ but $E\left(t_{1}\right)=E\left(t_{2}+f\right)$. Here $\Delta t$ was respectively equal to $60,100,65,000$ and 60,000 in the ScF, LF and SF cases.

\section{Diversity}

We used the "true diversity index" of order two (Jost, 2006) to measure the phenotypic and genotypic diversity at every 
iteration $t$ of the $\mathrm{CA}$ :

$$
{ }^{2} D_{p}(t)=1 / \sum_{k=1}^{5} \frac{N^{2}\left(s_{k}, t\right)}{N^{2}(t)}, \quad{ }^{2} D_{g}(t)=1 / \sum_{g=1}^{G(t)} \frac{N^{2}(g, t)}{N^{2}(t)},
$$

where $G(t)$ is the number of distinct genotypes at iteration $t$ and $N(g, t)$ is the number of cells sharing genotype $g$ at iteration $t$. Note that $N(t)=\sum_{k=1}^{5} N\left(s_{k}, t\right)=\sum_{g=1}^{G(t)} N(g, t)$.

\section{Homogeneous Test}

We also collected the most common genotype (MCG), i.e. the most frequently occurring one, starting at iteration $2,500,{ }^{1}$ then 102,500 and again about every 100,000 steps until iteration 500,000, thus creating 6 sampling points. All MCGs collected in that way were exported and tested alone in homogeneous conditions, i.e. where all cells were initialized with that genotype and no mutations could occur. Since each collected MCG from any one of the four fluctuating environments was cross-tested again in all four environments, we performed a total of $4 \times 50 \times 6 \times 4=4800$ runs. The maximum duration of these runs was set to 60,000 iterations. Sometimes the genotype was not adapted to the environment and all living cells went extinct (i.e. turned into the decay or quiescent state) before the end of the simulation. We considered a homogeneity test to be successful if living cells did not all go extinct before 60,000 iterations. In the Results section, we report the success rate of those simulations, along with statistics on the last iterations of the failed runs.

\section{Phenotypic Disturbance in the Homogeneous Test}

In HetCA, to survive on the long term genotypes must regularly "release" cells by transforming them into quiescent cells without genotypes. This generates patterns and cycles easy to observe in homogeneous simulations where a single genotype is tested (Fig. 2a). It is also observable, although with greater difficulty, in standard heterogeneous HetCA simulations (Fig. 2b). This is why, to characterize phenotypes we found it useful to measure these cycles as well as their irregularities. At every iteration $t>8$ of the homogeneous genotype test, we compared the sequence of states $s(c, t)$ and $s(c, t-1)$ of each cell $c$ to its anterior sequences during the previous 8 iterations of the CA. We assessed whether this sequence of two states was repeated and with what periodicity $p=\min \{p \in[2,7]\}$, i.e. such that $(s(c, t), s(c, t-1))=(s(c, t-p), s(c, t-p-1))$. We used a sequence of two states because if the genotype of a cell adopts a stable strategy, i.e. repeats a sequence of states, this sequence must contain a minimum of two states in order to ensure the survival of the genotype - the quiescent state and one of the living states. We chose to limit the comparison to

\footnotetext{
${ }^{1}$ It was shown by Medernach et al. (2015) that during the initial iterations of HetCA the MCGs were unlikely to exhibit any viable survival strategy.
}

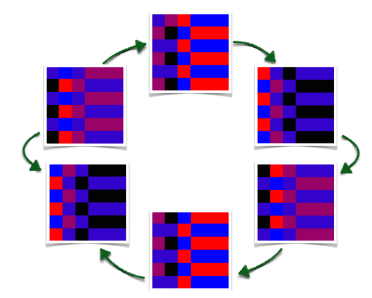

(a)

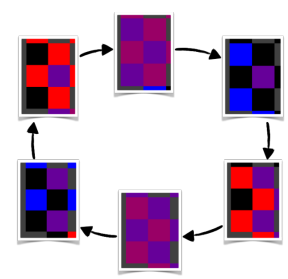

(b)
Figure 2: Examples of 6-step survival strategies: (a) Genotype extracted from a HetCA simulation in a stable environment (SE) with random homogeneous initialization. (b) Genotype produced by short-cycle fluctuations $(\mathrm{ScF})$.

the 8 previous iterations in order to reduce the computational cost and because the limit of 7 consecutive live iterations before decay involves, for a successful regular phenotype, a maximum periodicity of 7 iterations for the quiescent state. We performed this measure only if there was at least one living state and no decay among the last two states. For each iteration $t$ of the $\mathrm{CA}$, we reported the phenotypic disturbance

$$
P(t)=\sum_{p=1}^{7}\left|\frac{N(p, t)}{N(t)}-\frac{N(p, t-1)}{N(t-1)}\right|
$$

where $N(p, t)$ is the total number of cell that had periodicity $p$ at iteration $t$. This measure is rough but interesting because, unlike phenotypic differences, it is not directly based on states and therefore is less likely to be correlated to a state's probability of propagation.

\section{Results}

\section{Genotype Size and Genotype Mutations}

In the evolution of genotype size under the 4 types of environmental fluctuations (Fig. 3), we notice that the imposed size limit of 50 program statements tends to blur the differences between the different scenarios since most simulations converge to this limit. Yet, $\mathrm{ScF}$ clearly restricts the size of the genotypes more severely than SE, LF and SF, while these other conditions do not appear to influence genotype size. This size reduction by $\mathrm{ScF}$ could be a way to increase the impact of genotypic mutations on the phenotype. This is because, even though the number of statements potentially affected by mutations in LGP increases proportionally with genotype size, hence could have a larger effect on the phenotype, there can also be a "buffer effect" brought by information redundancy in longer genotypes, which would in fact stabilize the phenotype. Hence, mutations in more compact genomes might end up being more impactful ${ }^{2}$.

Observing the amount of mutations separating the current MCG from individuals created during initialization (Fig. 4),

\footnotetext{
${ }^{2}$ This assessment was supported by an analysis of the effective length of the most common individuals (not reported here).
} 


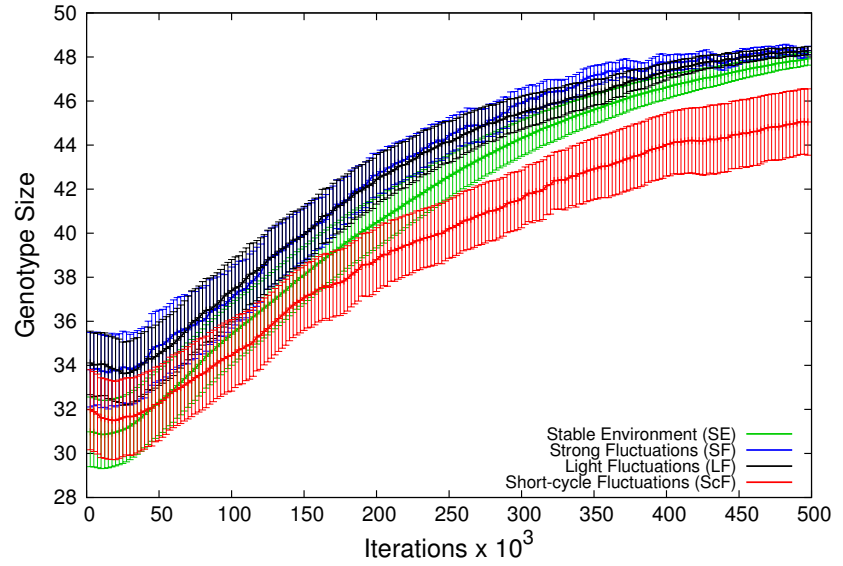

Figure 3: Evolution of genotype sizes (average \pm SEM) in number of LGP statements.

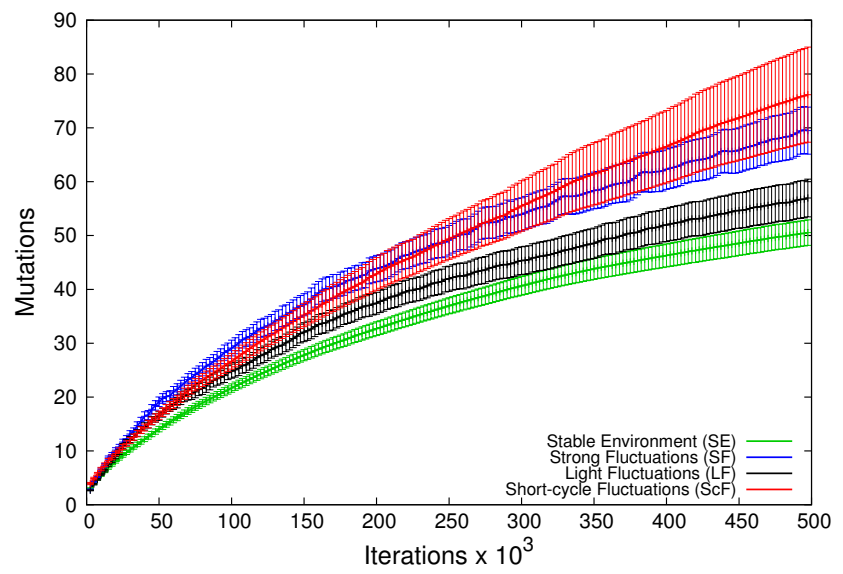

Figure 4: Evolution of number of ancestral mutations (average \pm SEM) involved in producing the current MCG.

it is not surprising to see that more mutations are selected for when environmental fluctuations are introduced. Their number also seem to depend more on the strength of these fluctuations (i.e. the contrast between two successive propagation probability patterns $E$ ) than on their periodicity. We also note that the proximity of $\mathrm{SF}$ and $\mathrm{ScF}$ indicates that their differences in size are not explained by differences in the number of selected mutations.

\section{Phenotypic Comparison}

In the phenotypic comparison $\sigma\left(t_{1}, t_{2}\right)$ between dissimilar environments, i.e. at $t_{1}$ and $t_{2}=t_{1}-\Delta t$ such that $E\left(t_{1}\right) \neq E\left(t_{2}\right)$ but $E\left(t_{1}\right)=E\left(t_{2}+f\right)$ (Fig. 5), we observe that the impact of environmental fluctuations decreases quickly for ScF while it remains very high for other types of environmental fluctuations. The phenotypic difference of $\mathrm{ScF}$ also remains most of the time lower than the phenotypic differences of the SE. This suggests the selection of a single phenotype, robust in both environments. Looking at $\sigma$ be-

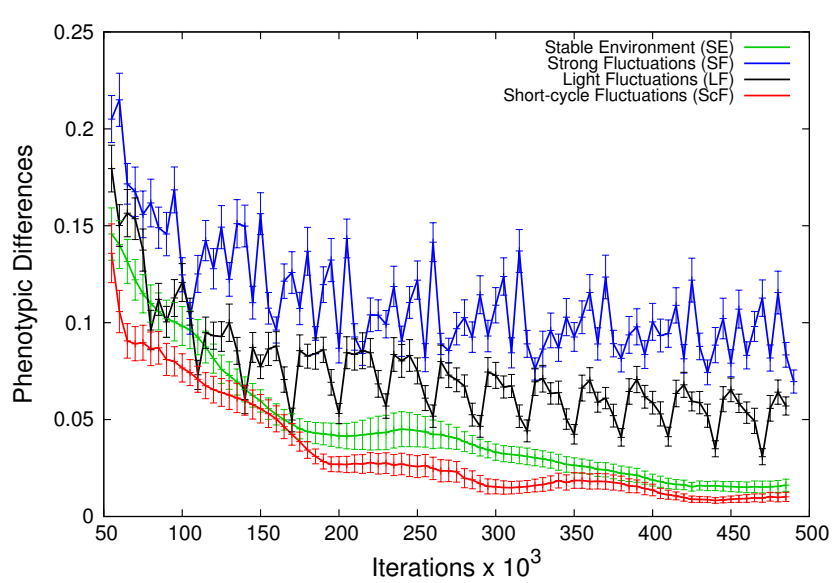

Figure 5: Evolution of phenotypic comparison function $\sigma$ (average \pm SEM) between dissimilar environments.

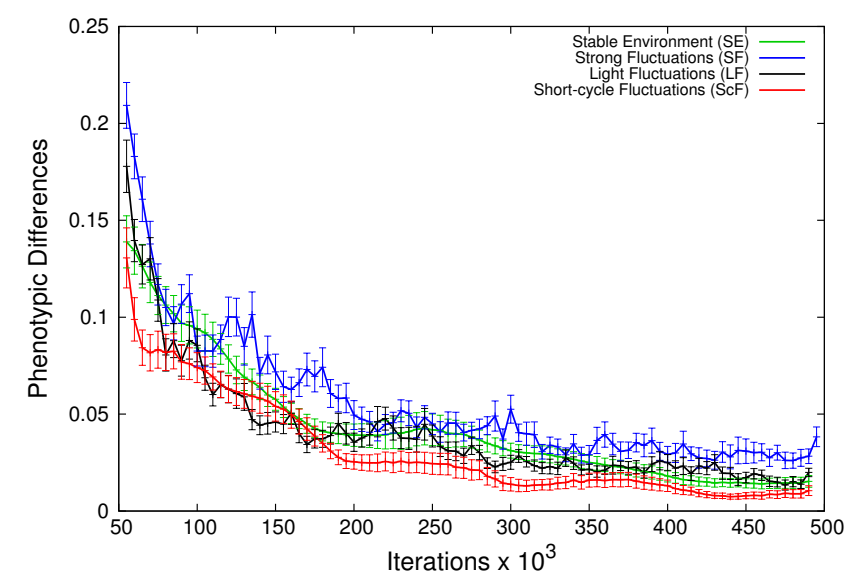

Figure 6: Evolution of phenotypic comparison function $\sigma$ (average \pm SEM) between similar environments.

tween similar environments, i.e. such that $E\left(t_{1}\right)=E\left(t_{2}\right)$ (Fig. 6), we note that phenotypic differences in LF and SF are much lower than in Fig. 5.

\section{Phenotypic and Genotypic Diversity}

Figures 7 and 8 depict the average phenotypic and genotypic diversities, ${ }^{2} D_{p}$ and ${ }^{2} D_{g}$. The generally low phenotypic diversity of $\mathrm{ScF}$ suggests the existence in this configuration of a dominant phenotype, which remains rather stable over time, whereas the relatively high phenotypic diversity of LF and SF combined with their relatively low genotypic diversity might suggest the existence of strong phenotypic selection, hence some form of plasticity.

\section{Success Rates of the Homogeneous Test}

Success rates of genotypes in different homogeneous simulations are reported in Fig. 9 using a normal approximation with a $95 \%$ confidence interval. The fact that SE offers the lowest challenge is not surprising. Similarly, the fact that SF 


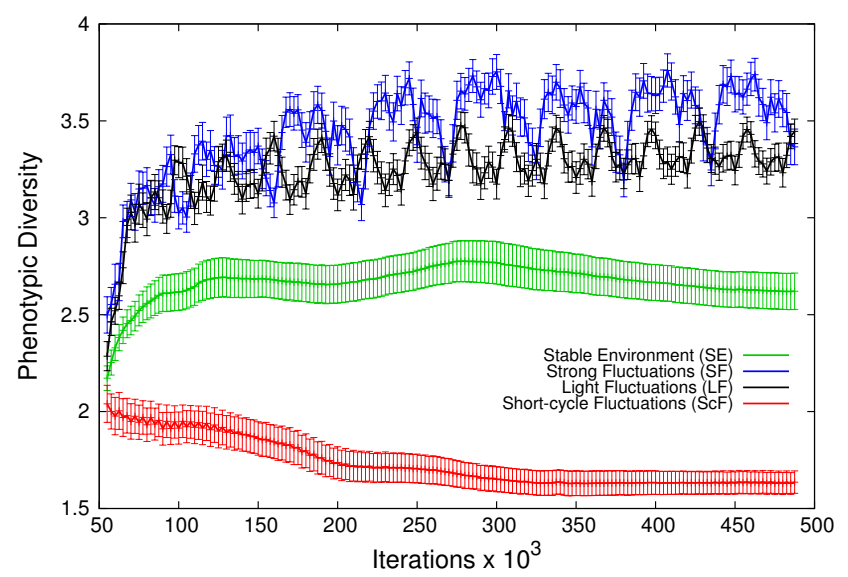

Figure 7: Evolution of phenotypic diversity (average \pm SEM).

is the least conducive to success is also expected. A comparison of the levels of difficulty between $\mathrm{LF}$ and $\mathrm{ScF}$ is less clear, however, since $\mathrm{ScF}$ performs significantly better in its own settings while on the contrary all other tested fluctuations are slightly more efficient in LF. It is also noteworthy that individuals from LF and SF seem relatively robust in various environmental configurations, while those from $\mathrm{ScF}$ seem fragile outside the environmental conditions in which they evolved. Moreover, among genotypes collected from iteration 102,500 , these same individuals are the only ones that do not reach a $100 \%$ survival ratio in a stable environment.

\section{Ending Iteration}

Displaying the last iterations reached by living cells of homogeneous runs, with genotypes collected at iteration 100,000 and 500,000 (Fig. 10), we see that the ScF genotype failures are concentrated around iteration 15,000 in LF homogeneous tests and 25,000 in SF homogeneous tests. This corresponds for these two configurations to the first environment for which $K\left(s_{3}\right)=0$, whereas $K\left(s_{3}\right)=1$ for all distributions $E$ in ScF. But we also see that some of the genotypes from $\mathrm{ScF}$ fail during the early iterations of the homogeneous test regardless of the environmental fluctuations, including SE and ScF. This might imply that the ecosystem resulting from the evolutionary history of the individuals plays a key role in their ability to survive in ScF.

\section{Analysis \\ Environmental Transitions}

Figure 11 visualizes the typical transitions between environments in different homogeneous runs. To compute these transitions we averaged the phenotypic disturbance $P$ over iterations $[t-40, t+40]$ for all $t \geq 5,000$ and $t \in T_{r}$, where $T_{r}$ is the sequence of iterations of run $r$ at which a transition between environments effectively occurred, i.e. for which $E(t) \neq E(t+1)$. Figure 11d shows the average transitions

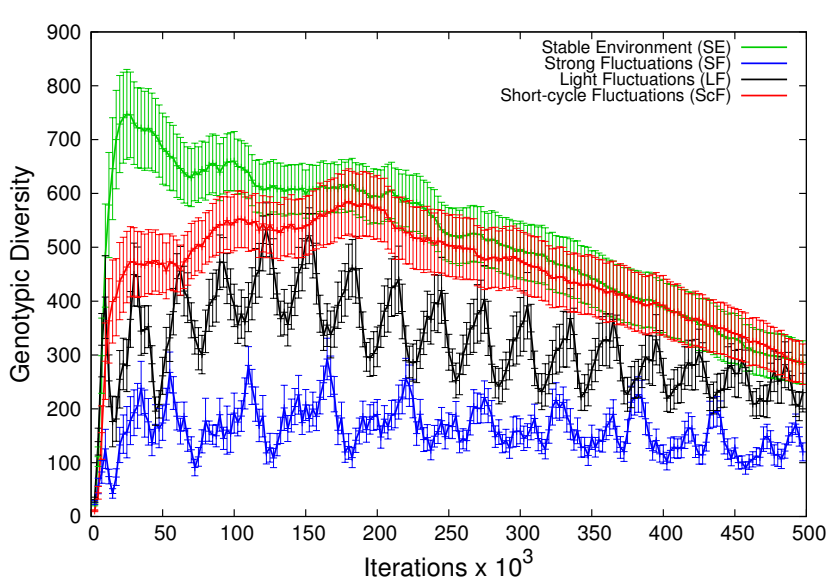

Figure 8: Evolution of genotypic diversity (average \pm SEM).

of ScF genotypes collected at the 6 aforementioned time steps $\{2,500,102,500, \ldots, 500,000\}$ and subjected to a homogeneous $\mathrm{ScF}$ test. It shows that phenotypes corresponding to genotypes collected later in the evolutionary process are less sensitive to environmental fluctuations. Conversely, as reported in Fig. 11b, the phenotypes of genotypes from SF keep the same high sensitivity regardless of the iteration at which they were collected. Finally, Fig. 11a and 11c compare the average transitions in homogeneous $\mathrm{ScF}$ and SF tests with genotypes collected at iteration 500,000 from the four different configurations. Again, it can be observed there that the phenotype of $\mathrm{ScF}$ is much more stable than the others in its original environment, but is at the same time very sensitive to transitions in SF.

\section{Phenotypic Diversity}

The phenotypic diversity measured in Fig. 7 can also be observed by visual inspection of the $\mathrm{CA}$ as displayed in the screenshots of Fig. 12. First, LF and SF are visibly different from ScF. These two groups diverge significantly in texture and also clearly differ from SE. Individuals from $\mathrm{ScF}$ seem to produce stable and robust phenotypes in any environment encountered within a $\mathrm{ScF}$ scheme. Their adaptations appear to be essentially created by genotypic mutations. They are also very dependent on their original ecosystem, sometimes distinctively so (Fig. 13), and as a consequence they are not robust in other types of fluctuations, where the effect of mutations is probably enhanced by the reduced size of the genotypes, as mentioned previously. By contrast, individuals evolved within LF or SF have high phenotypic diversity, and it seems likely that phenotypic selection occurs despite their lower genotypic diversity.

\section{Conclusions}

The phenotypic selection observed in this series of simulations evokes the multilevel selection model described by Jablonka et al. (2014). Among the three tested envi- 

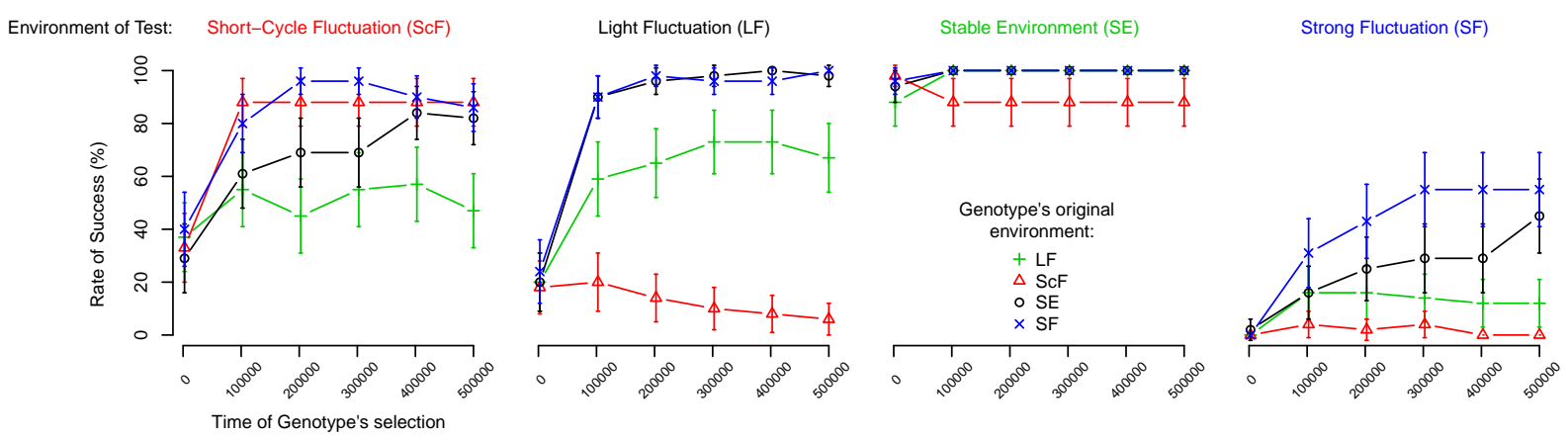

Figure 9: Evolution of success rate of genotypes (average \pm SEM) in homogeneous tests.

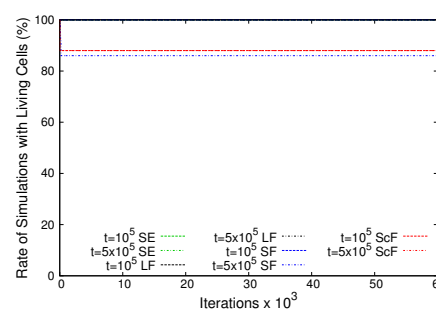

(a) $\mathrm{SE}$

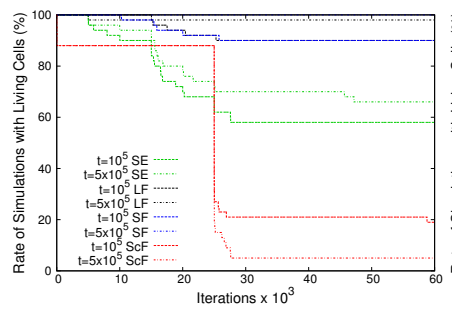

(c) LF

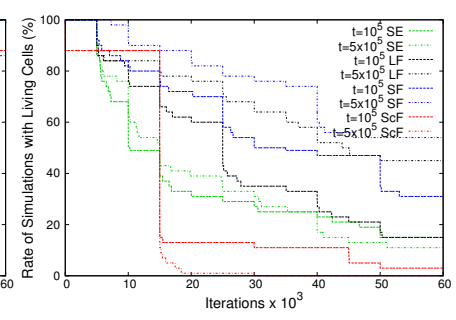

(b) $\mathrm{SF}$

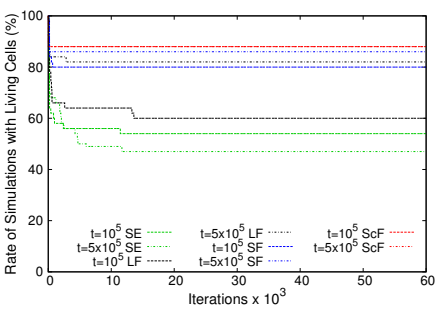

(d) $\mathrm{ScF}$

Figure 10: Last iterations reached by living cells of homogeneous runs of genotypes from iterations 100,000 and 500,000.

ronmental fluctuations, the LF and SF simulations are displaying the greatest similarity with this model. In $\mathrm{ScF}$, the best evolutionary strategy seems to involve small genotypes, which could be favored for their capacity to maximize the phenotypic impact of mutations. Furthermore, the inability of most $\mathrm{ScF}$ individuals to survive outside of the ecosystem resulting from their evolutionary history is reminiscent of the impossibility of saving species solely by preserving their DNA, as claimed in Jablonka et al. (2014): "You would have to reconstruct the community, and often these communities are very old, with historical memories that are stored in their epigenetic and behavioral systems. These are part of their 'identity,' part of their stability. You cannot freeze these memories: they have to be maintained and transmitted through use, so you cannot reconstruct the communities from their component parts." (ibid., p. 363). However, these experiments alone could not determine whether the main distinguishing feature between LF and SF on the one hand
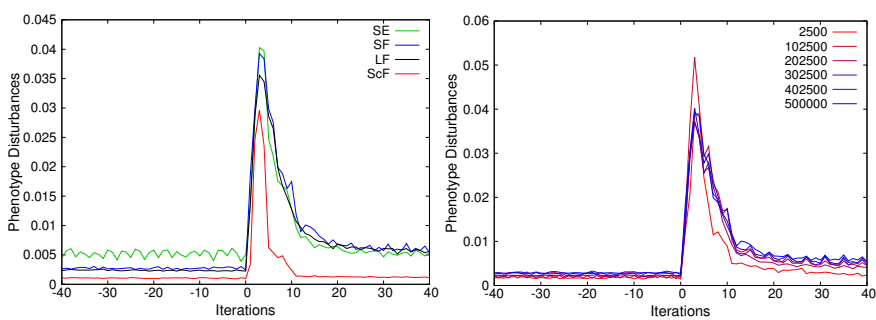

(a) SF: genomes from $t=5 \times 10^{5}$

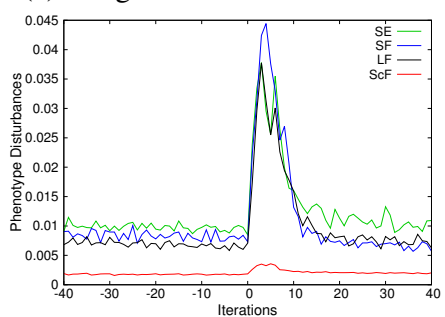

(b) SF: genomes from SF

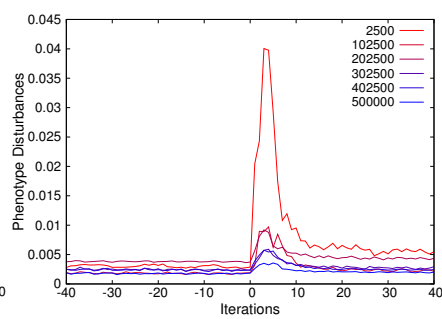

(c) ScF: genomes from $t=5 \times 10^{5}$

(d) $\mathrm{ScF}$ : genomes from $\mathrm{ScF}$

Figure 11: Phenotypic disturbance: Average transition between environments in different types of homogeneous tests.

and $\mathrm{ScF}$ on the other hand was the duration of the environmental cycles or the number of environmental types. Further investigation is needed.

\section{Acknowledgments}

Funding for this work was provided by the Science Foundation Ireland and the ERC Advanced Grant EPNet \#340828. Some of the simulations were run on the MareNostrum supercomputer of the Barcelona Supercomputing Center.

\section{References}

Bredeche, N. and Montanier, J.-M. (2012). Environmentdriven open-ended evolution with a population of autonomous robots. In Evolving Phys. Systems Workshop.

Floreano, D. and Urzelai, J. (2000). Evolutionary robots with on-line self-organization and behavioral fitness. Neural Networks, 13(4):431-443. 


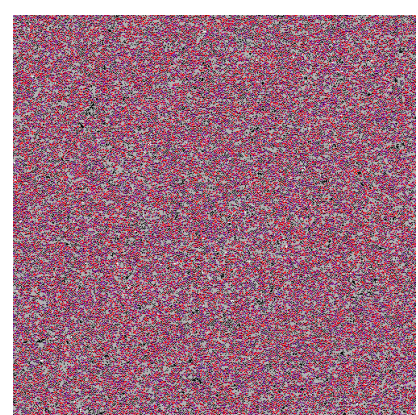

SE

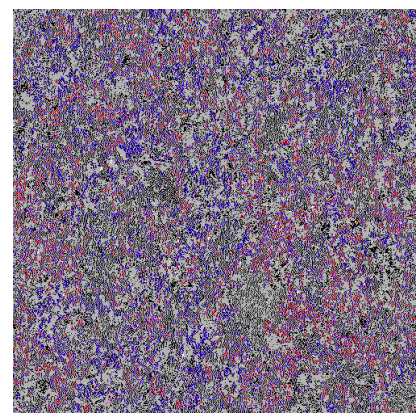

LF

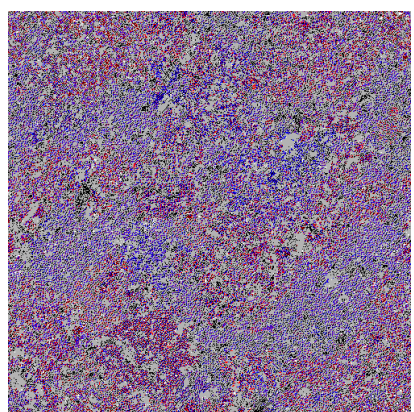

SF

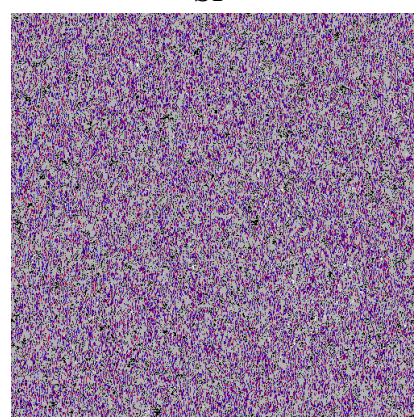

$\mathrm{ScF}$

Figure 12: Screenshots of the CA. Grid state distribution (phenotype) at iteration 495,000 in the four different configurations. Each cell state is represented by a different color. Black and grey represent cells in the decay and quiescent states, respectively. Shades of blue, red and purple represent the living states.

Heard, E. and Martienssen, R. A. (2014). Transgenerational epigenetic inheritance: myths and mechanisms. Cell, 157(1):95-109.

Jablonka, E., Lamb, M. J., and Zeligowski, A. (2014). Evolution in Four Dimensions, revised edition: Genetic, Epigenetic, Behavioral, and Symbolic Variation in the History of Life. MIT press.

Jost, L. (2006). Entropy and diversity. Oikos, 113(2):363375.

Lachmann, M. and Jablonka, E. (1996). The inheritance of phenotypes: an adaptation to fluctuating environments. Journal of theoretical biology, 181(1):1-9.

Laland, K., Matthews, B., and Feldman, M. W. (2016). An introduction to niche construction theory. Evolutionary Ecology, 30(2):191-202.

Levins, R. (1968). Evolution in changing environments: some theoretical explorations. Princeton Univ Press.

Lipson, H., Pollack, J. B., and Suh, N. P. (2002). On the origin of modular variation. Evolution, 56(8):1549-1556.

Lloyd, E. (2012). Units and levels of selection. In Zalta, E. N., editor, The Stanford Encyclopedia of Philosophy. Stanford University, spring 2012 edition.

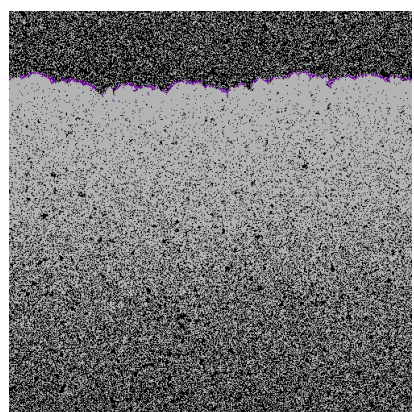

$t=100,000$

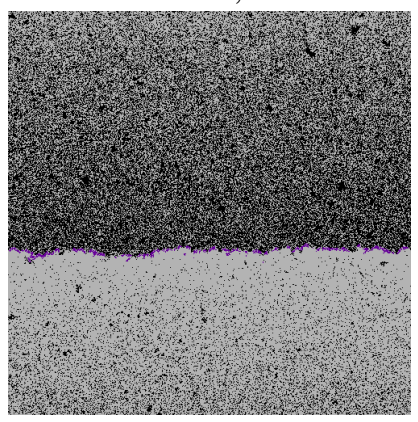

$t=400,000$

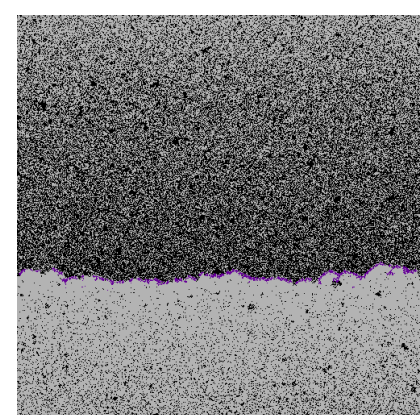

$t=200,000$

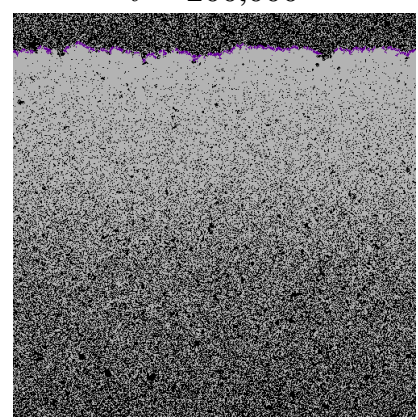

$t=500,000$
Figure 13: Original ScF simulation: a distinctive "wavy" phenotype, very stable over time, produces genotypes that fail in the early iterations of the homogeneous test.

Medernach, D., Fitzgerald, J., Carrignon, S., and Rya, C. (2015). Evolutionary progress in heterogenous cellular automata (hetca). In Proceedings of the European Conference on Artificial Life 2015 (ECAL 2015), volume 13, pages 512-519.

Medernach, D., Kowaliw, T., Ryan, C., and Doursat, R. (2013). Long-term evolutionary dynamics in heterogeneous cellular automata. In Proceedings of the 15th annual conference on Genetic and evolutionary computation, pages 231-238. ACM.

Müller, G. B. (2007). Evo-devo: extending the evolutionary synthesis. Nature Reviews Genetics, 8(12):943-949.

Okasha, S. (2006). Evolution and the levels of selection, volume 16. Clarendon Press Oxford.

Shanahan, T. (2012). Evolutionary progress: conceptual issues. eLS.

West-Eberhard, M. J. (2005). Developmental plasticity and the origin of species differences. PNAS, 102(suppl 1):6543-6549.

Yu, T. (2007). Program evolvability under environmental variations and neutrality. In Proceedings of the 9th annual conference companion on Genetic and evolutionary computation, pages 2973-2978. ACM. 